\title{
An Improved Neuron Segmentation Model for Crack Detection - Image Segmentation Model
}

\author{
Ming Hao ${ }^{1}$, Caifeng Lu ${ }^{1}$, Guoqing Wang ${ }^{2}$, Wei Wang ${ }^{1}$ \\ ${ }^{1}$ State Key Laboratory for Geomechanics and Deep Underground Engineering, China University of \\ Mining and Technology, Xuzhou, Jiangsu 221116, China \\ ${ }^{2}$ School of Information and Control Engineering, China University of Mining and Technology, Xuzhou \\ 221116, China \\ E-mails:15950687063@163.com lucaifeng@cumt.edu.cnwangguoqingcumt@hotmail.com \\ wangweioh@163.com
}

\begin{abstract}
It is still very challenging to establish a unified and robust framework to perform accurate and complete crack extraction from images with cluttered background, various morphological differences and even with shadow influence. In this paper, an improved neuron segmentation model with two stages is proposed for crack segmentation. Firstly, a robust crack indicator function is designed based on local directional filtering; it makes up for the traditional function based on hessian matrix, which is resulting in problem of local structure discontinuities. After obtaining the indicator function, the crack detection is performed in an integrated mode; it is incorporating the automated directional region growing without manual intervention by adopting level sets; then efficient and complete crack segmentation is realized by iterative contour evolution. The performance of the proposed model is demonstrated by experiments on three kinds of grouped crack sample images and the quantitative evaluation. We also argue that the proposed model is applicable for biomedical image segmentation.
\end{abstract}

Keywords: Crack detection, crack indicator function, local directional filtering, directional region growing, level set.

\section{Introduction}

Crack, one of the most common phenomenon in concrete infrastructure, has resulted in many reported unexpected accidents $[1,2]$ that are related to insufficient inspection and condition assessment by manual operation [3]. Typically, condition assessment is performed manually with the help of certified inspectors and/or structural engineers, either at regular intervals (routine inspection) or after unexpected disasters (post-disaster inspection). However, continuous inspection and assessment on the 
physical and functional conditions of existing civil infrastructure is essential to ensure the safety and serviceability for preventing such kinds of accidents, but is impossible for typically manual operation. Computer vision-based modules, an integral component of modern Structural Health Monitoring (SHM) framework [4], has been adopted to automate the process of defect and damage detection (especially for common crack detection) due to the availability of low cost, high quality and easyto-use visual sensing technologies (e.g., digital cameras).

Inspired by the review of the existing creack detection methods in $[5,6]$, we rigorously classify the relevant methods broadly as edge detection, segmentation and percolation, local information-based method, machine learning methods, region growing methods, and other techniques. Various edge detection algorithms are compared in [7] and the Haar Wavelet method is declared to be the most reliable among them for the purpose of crack detection. However, the performance of edge detection algorithms on noisy infrastructural image is remained to be questionable; it is the same case with morphological operation based methods [8]. Scable local percolation-based image processing techniques are tried in [9] to get the efficient and accurate detection result even for large surface images [10]. Some other local methods such as statistical filter [11], morphological filter [12], Hessian matrix-based filter [13] and wavelets $[14,15]$ are also applied successfully when the texture of surfaces is fine grained; the performance will deteriorate significantly and sharply $[16,17]$ when surface is coarse- grained and cracks are not continuous. The classical Principal Component Analysis (PCA) based algorithm [18] is adopted to detect cracks on a bridge surface, in which case the accuracy of results varies with camera pose and capturing distance where images are taken. Prasanna et al. [19] designed a histogram-based classification algorithm and used it along with Support Vector Machines (SVM) to detect cracks on a concrete deck surface; the result on real bridge data highlighted the need for improving the practical prediction accuracy. Similarly, an automatic clustering mehtod [20] is proposed for segmentation based on Canny and K-Means to achieve greater accuracy of crack detection under various enviromental conditions at a greater processing speed.

To extract the inconspicuous cracks embedded in cluttered background, a number of region-growing based methods have been proposed. Among them, the minimal path-based methods [16, 21-25], which appear to be most prevalent in recent literature, consider a crack as a path of minimal length in a certain metric and then convert the crack detection problem to that of finding the minimal path. Similarly, the cracks with complicated geometrical changes such as branches and curves could also be coped in [26-28]. In such scenarios, seed points are chosen, firslty, by manual or automatic way, and then the connectivity process with the optimal set of seeds is fulfilled using a shortest path algorithm [29] by suitably selecting the weights on the graph edges. Fast and accurate detection is possible using the above mentioned region growing approaches if the crack structure is morphologically simple and the image noise level is low. It is extremely difficult to cope with the complicated topology changes of cracks such as disconnections and closed substructures, and only [16, 23] are able to exract cracks with arbitrary topology. 
There are also some other distinctive methods such as 3D reconstruction [30-32], in which the researchers combine image-based 3D scene constructions with other techniques to obtain the depth perception that a 2D image lacks. The general procedure of 3D methods refers to firstly, recovering the 3D structure of an object usig several images captured from different views, and then the cracks could be segmented by preset morphological filters. The key idea is that the structural element size of the morphological operator can be adaptively adjusted according to some parameters computed from the 3D model and the desired crack thickness. However, this effective methods are not appropriate for the common single-view images.

The paper focuses on designing the neuron-model segmentation algorithms to realize the crack detection with cluttered background after finding the great similarity between the neural image and the traditional crack images of civil infrastructure. As shown in Fig. 1, it could be concluded that both the confocal microscopy image [33] and the concrete image, in general, degraded by low signal to noise ratio and non uniform illumination of the neurites (topological crosspoints). Topologically speaking, both parts of Fig. 1 represent that a neuron (crosspoints) resembles a tree, with multiple filamentous branches emerging from a single or multiple cell parts. The circled part represents the branches growing source points which indicate the similarity between the two different kinds of images. The image on Fig. 1a, similar with the one in [33], is generated by the authors of this paper based on the algorithm proposed in [33].

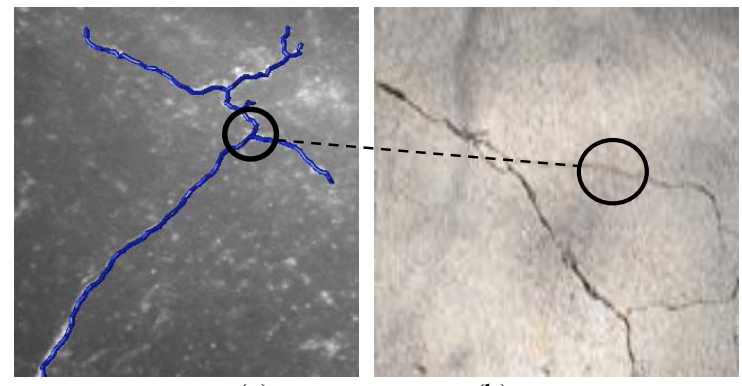

(a)

(b)

Fig. 1. A reconstructed Drosophila neuron image (a); a selected complicated-cracked concreted image with shadow (b)

The classical neuron-model segmentation schemes could be broadly categorized into two basic approaches. The first set is based on user-defined/automatically detected initial seed points to realize the tracing procedure. The second one avoids seed initialization and performs segmentation globally.

The manual seed selection of the first approach has the advantage that the segmentation region is identified as prior knowledge by an expert. A l-K of a hi et al. [34] tries the medial response of multiple directional templaets to determine the direction to generate robust seed points along the neuron medial axis. The local growing method shows good performance with high-quality images, but requires continuity in the neuron branches for reliable segmentation. As for the automatic selection model, the branches growing and connection is done [27, 28] between the optimal set of seeds by finding the shortest path [29]. The disadvantage is the same: 
the final result is greatly associated with the simplicity and quality of the original crack images. What's more, the segmentation accuracy is sometimes degraded if suboptimal set of points is chosen. On the other hand, the connectivity analysis between the seeds assumes uniform intensity distribution.

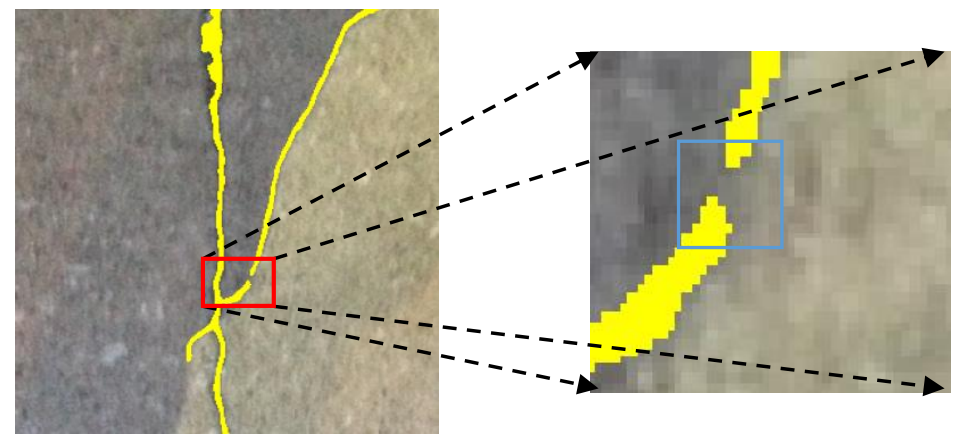

Fig. 2. The segmentation result suffers from background non-uniformity, including gap that leads to break in the segmented crack structure processed by Tree2Tree [40]. The effect region is bounded by red dashed box, magnified here for improved viewing

In contrast to the seed based local techniques, the typical global methods rely on a four stage processing pipeline-enhancement, segmentation, centerline detection and post processing. The region growing, representative one of global methods, is tried in $[35,36]$, and the segmentation has realized a centerline detection process [37, 38] followed by the further smoothing of the medial axis with spline fitting [39]. Benefiting from no human intervention, the initial segmentation, which may be easily affected by the existing noise and clutter, would heavily influence the final detection reuslt.

The paper proposes an improved neuron structure-based segmentation scheme to adress two primary issues existing in the above mentioned models. First, the proposed technique is suited to identify the multivariate crack distribution from the clutted images. Second, the neuron-like model is adept at handling the local structure discontinuities (as shown in Fig. 2 resulted from Tree2Tree [40]) resulting from imaging artifacts and uncontrolled existence of shadow effect. The proposed segmentation solution is designed under a variational framework driven by level sets [41], and the vital level set evolution is guided by minimizing a specifically set energy function [42].

The proposed model can be decomposed into two stages (as shown in Fig. 3). Initially, a robust vessel indicator function is computed to suggest the presence of a crack at each location of the digital image. This indicator function, along with the crack orientation, is then used to iteratively propagate a geometric active contour to segment the filamentous structure. Besides, the proposed method does not exert demand on initial set of seed points for segmentation when compared with that in [27-29]. Moreover, non-trivial post-segmentation analysis is not called for linking the disjoint segments, which is performed naturally by replacing the traditional hessian matrix based crack evidence generation with the proposed local directional filtering model. 


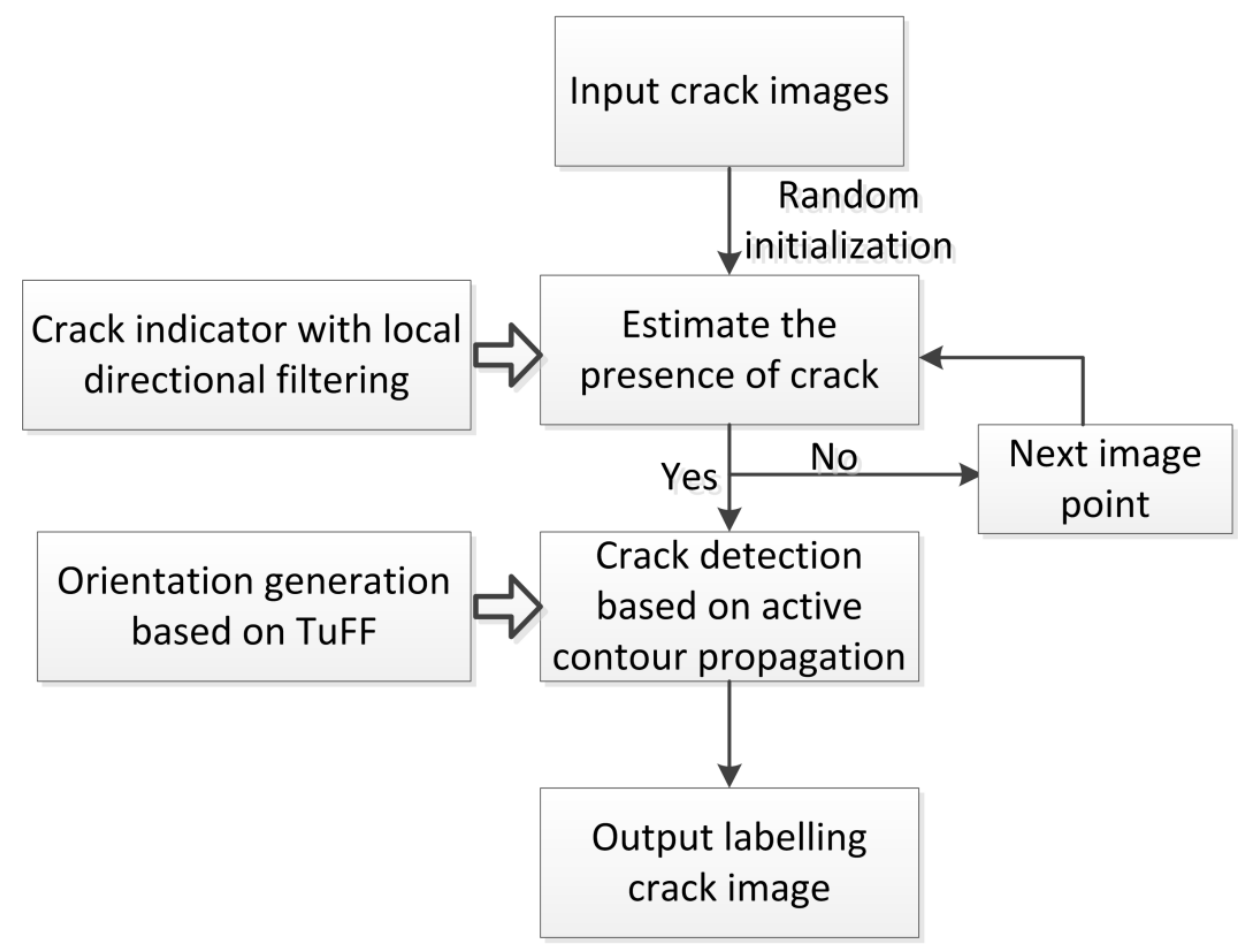

Fig. 3. Procedure of crack detection with the proposed model

The rest of the paper is organized as follows. Section 2 describes the problem formulation. In Section 3, we review the TuFF model and analyze its disadvantage in evidence generation design, followed by introducing the local directional filtering for model improvement in Section 4. Experiemnts and discussion are detaield in Section 4, and Section 5 gives the conclusion of the paper.

\section{Problem formulation}

Inspired by the problem of vascular structure segmentation and identification which has been well studied in the medical image processing literature, we propose a solution for robust identification of cracks on concrete surfaces by defining the active contour propagation with crack indicator function. The proposed model can be decomposed into two stages. Initially, a robust crack indicator function is computed to suggest the presence of a crack at each location of the digital image. This indicator function, along with the crack orientation direction (as formulated in Equation (12)) is then used to iteratively propagate a geometric active contour to segment the crack, which shows like filamentous structure. 


\section{Tubularity vector filed construction}

\subsection{Formulation of Tubularity flow field}

The key to the problem is to find effecitve method to segment filamentous structure, to which problem the structure segmentation is modelled as filamentous processing, and we focus on the method proposed by $\mathrm{Mukherje}$ e, B arry and A c t on [33]. This technique, called Tubularity Flow Field (TuFF), performs segmentation via propagation of a geometric active contour, which is implemented using level sets. In this formulation, a contour $C(x, y)$ propagates under the influence of two vector fields, called tubularity flow field (see Fig. 4). The mathematics governing the contour propagation can be written in terms of a PDE as follows:

$$
\frac{\partial \boldsymbol{C}}{\partial t}=\alpha_{1}<\mathbf{V}_{1}, \mathcal{N}>^{2} \mathcal{N}+\alpha_{2}<\mathbf{V}_{2}, \mathcal{N}>^{2} \mathcal{N},
$$

where $\mathcal{N}$ is a unit normal vector at each position on the contour $C(x, y)$. $\mathbf{V}_{\mathbf{1}}$ and $\mathbf{V}_{\mathbf{2}}$ are the axial and normal components of the tubularity flow field which signifies the crack orientation and the direction orthogonal to it (Fig. 4). With this formulation, the contour $C(x, y)$ undergoes motion such that it propagates both along the crack axis (due to $\mathbf{V}_{\mathbf{1}}$ ) and the crack thickness (due to $\mathbf{V}_{\mathbf{2}}$ ), the propagating speed being governed by the coefficients $\alpha_{1}, \alpha_{2}>0$.

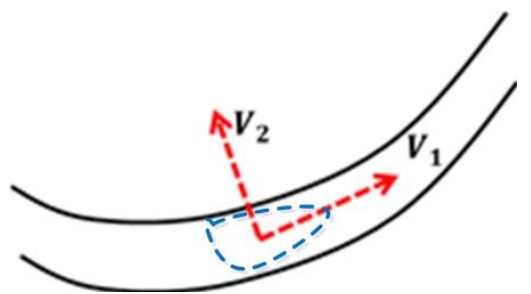

Fig. 4. Illustration of tubularity flow vector field (blue curve represents the iterated contour)

\subsection{Analysis of Hessian matrix based tubularity evidence generation in TuFF}

In [33], the authors have illustrated the effects $\alpha_{1}, \alpha_{2}$ on curve evolution. A popular choice for the directional coefficients is $\alpha_{1}=\alpha_{2}=\mathcal{R}(x, y)$. $\mathcal{R}(x, y)$ is a tubularity indicator function which assumes a high scalar value $(\cong 1)$ at locations, where a tubular structure is presented, and a low value $(\cong 0)$ when such a structure is absent. Such choice ensures that propagating active contour comes to a halt when the magnitude of tubularity indicator function diminishes. The design of $\mathcal{R}(x, y)$ in [33] is realized based on Hessian matrix analysis proposed by Frangi (see [2]).

The specific definition of hessian matrix for image $f(x, y)$ at position $(x, y)$ and scale $\sigma$ is the square matrix as follows:

$$
\begin{gathered}
H_{\sigma}(\boldsymbol{x}, \boldsymbol{y})=[h]_{i, j}(1 \leq i, j \leq 2),(\boldsymbol{x}, \boldsymbol{y}) \in \boldsymbol{\Omega}, \\
h_{i, j}=\frac{\partial^{2} G(\sigma)}{\partial x_{i} \partial x_{j}} * f(\boldsymbol{x}, \boldsymbol{y}),
\end{gathered}
$$

where $G(\sigma)$ represents the zero mean normalized Gaussian kernel with variance $\sigma^{2}$. Since the crack is represented by different intensity distribution from the background, one can analyze the scale space hessian matrix to obtain evidence of tubularity at a 
particular image position. However, due to the fact that the crack structures are modelled as piecewise rigid templates during hessian matrix construction, such local crack detection methods are less proficient in detecting complex crack geometries such as junctions and filament bends, thus resulting in local structure discontinuities.

To solve such a problem, a specialized local attraction force is designed to naturally line the fragmented structures. Despite its effectiveness in connecting discontinuities, it is rather time consuming by building a variational framework. Besides, such a model is not easy to transfer across images with general parameters definition.

To combat these issues, a solution is proposed by designing evidence filters for tubularity structure detection. By incorporating such a model for $\mathcal{R}(x, y)$, an improved and more efficient TuFF model for crack detection is realized.

\section{Improved TuFF with local directional filtering}

\subsection{Crack indicator function formulation with local directional filtering}

The principle of detecting crack structures from digital images can be interpreted from a template matching perspective [45]. An oriented crack template can be obtained by steering a second order Gaussian derivative filter to produce a filter bank of oriented local crack templates. The hessian matrix based template for filtering could be referenced from Fig. 5b. Generating the evidence of a filamentous structure is then carried out by filtering the image with this local crack template.

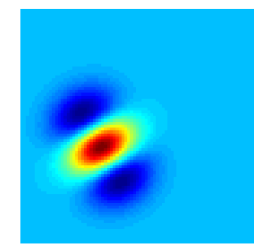

(a)

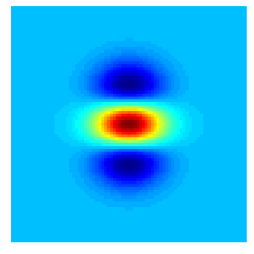

(b)

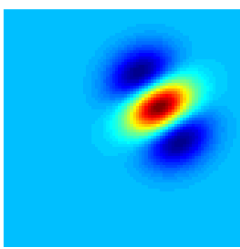

(c)

Fig. 5. Examples of a backward evidence filter, local evidence filter and forward evidence filter

To combat the issues that generating local structure discontinuities in traditional TuFF, a solution is proposed in terms of additional evidence filters. This local evidence filters (see Fig. 5a and c), also called forward and backward evidence filters $\mathcal{R}_{\mathrm{f}}$ and $\mathcal{R}_{\mathrm{b}}$ respectively, in conjunction with the hessian matrix based detector $\mathcal{R}_{\mathrm{d}}$ (Fig. 5b), the subscript "d" represents directional filter setup) provides evidence for the presence of a filamentous structure in a local neighborhood of the detection kernel.

The procedure for calculating the evidence of tubularity structure could be formulated as follows:

$$
\begin{gathered}
\mathcal{R}_{\mathrm{d}}^{*}=\max _{\theta} \mathcal{R}_{\mathrm{d}}((x, y), \theta ; \sigma), \\
\mathcal{R}_{\mathrm{d}}((x, y), \theta ; \sigma)=r_{\mathrm{d}}((x, y), \theta ; \sigma) \cdot f(x, y),
\end{gathered}
$$




$$
r_{\mathrm{d}}((x, y), \theta ; \sigma)=g_{x x} \cos ^{2} \theta+g_{y y} \sin ^{2} \theta+g_{x y} \sin 2 \theta,
$$

and $g_{x x}, g_{x y}, g_{y y}$ is the value of hessian matrix, which could be obtained by convolutional operation of pixel intensity with Gaussian kernel $\left(g(p ; \sigma)=\frac{1}{\sqrt{2 \pi} \sigma} e^{-\frac{x^{2}+y^{2}}{2 \sigma^{2}}}\right)$. Based on the framework for obtaining hessian matrix based evidence, the proposed local evidence filters could be formulated as follows:

$$
\begin{aligned}
& r_{\mathrm{f}}\left((x, y) ; \sigma, \varphi_{1}\right)=r_{\mathrm{d}}\left(x+d \cos \left(\theta+\varphi_{1}\right), y+d \sin \left(\theta+\varphi_{1}\right)\right), \\
& r_{\mathrm{b}}\left((x, y) ; \sigma, \varphi_{1}\right)=r_{\mathrm{d}}\left(x-d \cos \left(\theta+\varphi_{2}\right), y-d \sin \left(\theta+\varphi_{2}\right)\right) .
\end{aligned}
$$

Based on (5) and (7), (8), the response of local evidence filters for certain scale are:

$$
\begin{gathered}
\mathcal{R}_{\mathrm{f}}((x, y), \theta ; \sigma)=r_{\mathrm{f}}\left((x, y) ; \sigma, \varphi_{1}\right) \cdot f(x, y), \\
\mathcal{R}_{\mathrm{b}}((x, y), \theta ; \sigma)=r_{\mathrm{b}}\left((x, y) ; \sigma, \varphi_{1}\right) . f(x, y) .
\end{gathered}
$$

After figuring out the separate filter response, the crack indicator function could be obtained by superimposing the filter response of the detector and evidence kernels at multiple scales:

$$
\mathcal{R}(x, y)=\max _{\theta, \sigma} \mathcal{R}_{\mathrm{d}}+\max _{\varphi_{1}, \sigma} \mathcal{R}_{\mathrm{f}}+\max _{\varphi_{2}, \sigma} \mathcal{R}_{\mathrm{f}} .
$$

Based on the improved local directional filter processing, the indication results with methods in traditional TuFF and the improved one could be referenced from Fig. 6. Note that we use a set of oriented evidence filters for each orientation of the detector kernel, thus being able to incorporate crack evidence at multiple orientations. This technique is extremely powerful in detecting junction points and crack bends, thus providing a robust methodology for computing the crack indicator function.

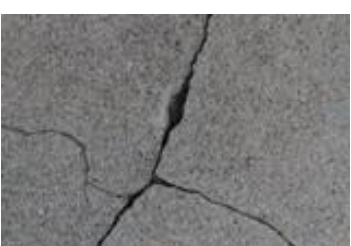

(a)

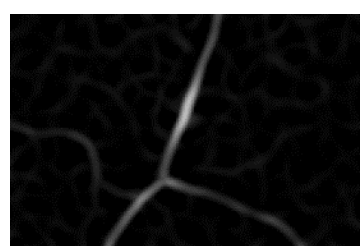

(b)

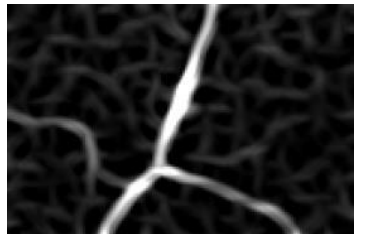

(c)

Fig. 6. Comparison of local filtering results with different methods: original image (a); crack indicator function via hessian matrix (b); crack indicator function via local directional filtering (c)

\subsection{Crack segmentation with improved TuFF}

Having defined the vessel indicator function, segmentation of the cracks can be performed using level set methods [46]. Level sets are a popular choice for implementing geometric curve motion and it has the advantage that the evolving contour can change its topology naturally. An embedding function $\phi(x, y, t)$ is used such that the contour $C(x, y)$ is represented by the zero level sets of $\phi$. The PDE in (1) reduces to the following expression which is solved using numerical techniques.

$$
\frac{\partial \phi(x, y, t)}{\partial t}=\mathcal{R}(x, y)\left(\left\langle\mathbf{V}_{\mathbf{1}},-\frac{\nabla \boldsymbol{\phi}}{|\boldsymbol{\nabla} \boldsymbol{\phi}|}\right\rangle^{2}+\left\langle\mathbf{V}_{\mathbf{2}},-\frac{\boldsymbol{\nabla} \boldsymbol{\phi}}{|\boldsymbol{\nabla} \boldsymbol{\phi}|}\right\rangle^{2}\right)|\boldsymbol{\nabla} \boldsymbol{\phi}| \text {. }
$$

To sovle the problem of crack detection modelled by Equation (12), it is essential to design the energe function such that it would encourage the curve 
propogation in the real crack lined region, while avoding the crack-like background parts.

According to [42], the segmentation of cracks is performed by minizing the defined energy function $\varepsilon(\varnothing)$, which could be mathmatically written as:

$$
\begin{array}{cc}
\varepsilon(\varnothing)=\varepsilon_{\mathrm{reg}}(\varnothing)+\varepsilon_{\text {evolve }}(\varnothing), \\
\varepsilon_{\mathrm{reg}}(\varnothing)=v_{1} \int_{\Omega}|\nabla \emptyset(\boldsymbol{x}, \boldsymbol{y})| \delta_{\epsilon}(\varnothing) d_{\boldsymbol{x}} d_{\boldsymbol{y}} \\
\left.(14) \quad \boldsymbol{D}_{\boldsymbol{i}}(\boldsymbol{x}, \boldsymbol{y}), \boldsymbol{n}(\boldsymbol{x}, \boldsymbol{y})\right\rangle^{2} H_{\epsilon}(\varnothing) d_{\boldsymbol{x}} d_{\boldsymbol{y}} .
\end{array}
$$

In the formulas, the energy function $\varepsilon_{\text {reg }}$, the regularizing part of which constrains the length of the original zero level set curve $\emptyset$, refers to the smoothness of the curve, while the $\varepsilon_{\text {evolve }}$ is set to realize the curve evolution repectively. The design of the positive scalar $v_{1}$ in (14) specifically contributes towards the smoothness of the zero level set $\varnothing$, the smaller of $v_{1}$ renders smaller and disjoint detected cracks in the final solution. The vector presence $n(x, y)=\frac{\nabla \emptyset(x, y)}{|\nabla \emptyset(x, y)|}$ in (15) denotes the inward normal unit vector against $\emptyset$, and the operational rule $\langle\cdot, \cdot\rangle$ is mathmatically defined as the Euclidean inner product of operator. The weighting parameter $\alpha_{i}(x, y)$ in (15) determines the contribution of the orthogonal and axial components of the improved TuFF part while conducting the curve evolution.

The details on methods designing to figure out the minimization problem could be referenced from [33].

\section{Experimental results and analysis}

In this section, we demonstrate the efficiency of the proposed model with different kinds of crack images and make analysis from different perspective. What's more, we further compare the segmentation performance to that of other two classical crack detection models.

\subsection{Evaluation of segmented results}

In this section, we domenstrate the visual performance of the proposed neuron segmentation model with three different kinds of characterized images including the images with single crack and multiple cracks, and also the ones with shadow which render great effect on the final detection result [56], and the effectivness is verified by comparing with other state-of-the art methods. All the test images share the same size of $115 \times 115$. The widths of cracks vary from 1 up to 5 pixels and the branches condition also differ shaprly while the backgrounds are coarse grained. The crack detection and the marked results are illustrated in Fig. 7; the ground-parts obtained by tracing the cracks manually on the original images are listed in the second column.

It could be observed that the crack segmentation using improved TuFF with local directional evidence performs far better than expectation without importing the fake information and other mis-detected parts. 


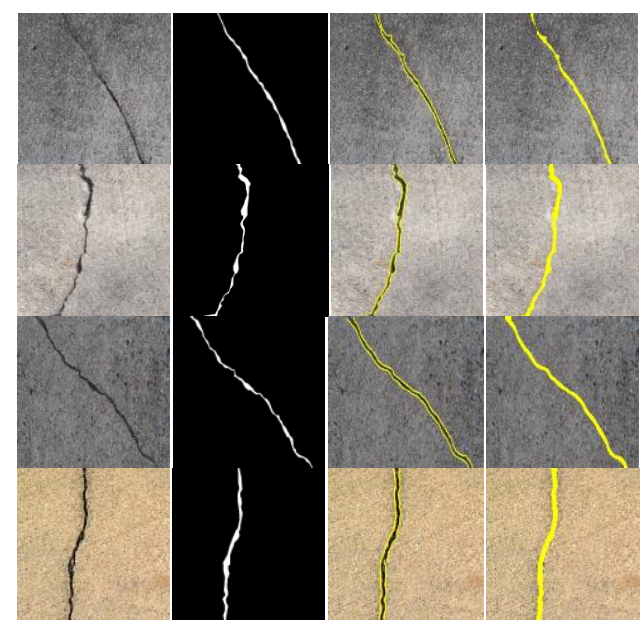

Group A: Images with single cracks

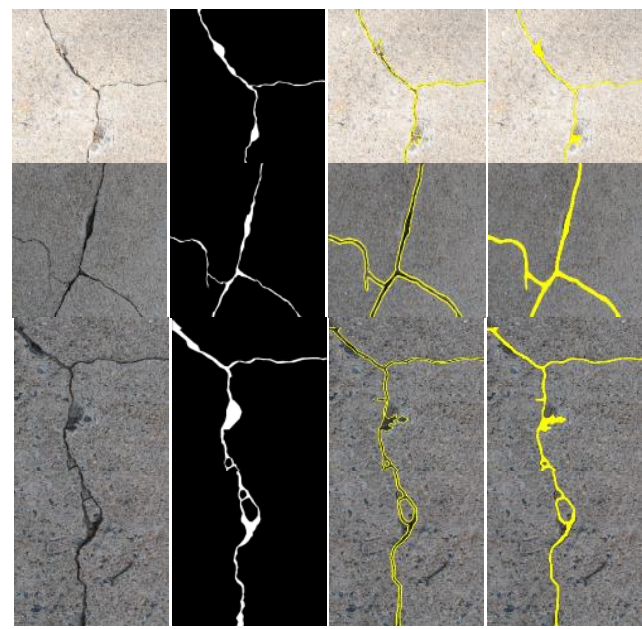

Group B: Images with branches

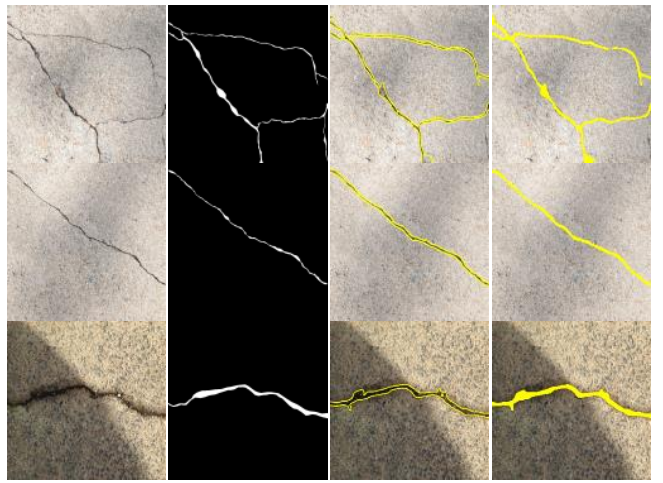

Group C: Images with shadows

Fig. 7. Crack detection results by the proposed algorithms. Group A demonstrates the result with only one crack; Group B demonstrates the result with multiple branches distribution; Group C displays the result with the influence of shadow 
On the other hand, to further quantify the detection performance, three newlydesigned measurements which reflect the efficiency of the particular methods are identified. The specific definition and corresponding results are as follows: number of over-estimated branches (Fig. 8a), number of missed branches (Fig. 8a) and finally the mean absolute error of the filled cracks with respect to the ground truth. The number of over-estimated and missed branches reflects the ability of handling the discontinuities of the morphology structure. The Mean Absolute Error (MAE) is obtained by formulation below (25). Define $\mathcal{P}=\left\{p_{1}, p_{2}, \cdots, p_{n}\right\}$ and $\mathcal{Q}=\left\{q_{1}, q_{2}, \cdots, q_{m}\right\}$, denote the coordinates set by a certain iteration, the specific mean absolute error between each segmented cracks detail is given by

$$
\mathrm{MAE}=\frac{1}{n} \sum_{i=1}^{n} \min _{j}\left|p_{i}-q_{j}\right|+\frac{1}{m} \sum_{i=1}^{m} \min _{k}\left|q_{i}-p_{k}\right|
$$

It should be claimed that $\forall j \in\{1,2, \cdots, m\}$ and $\forall k \in\{1,2, \cdots, n\}$.

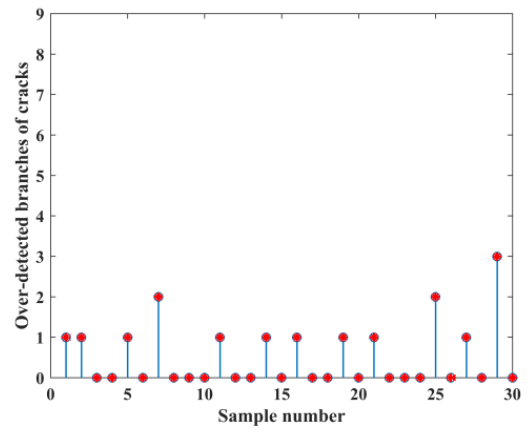

(a)

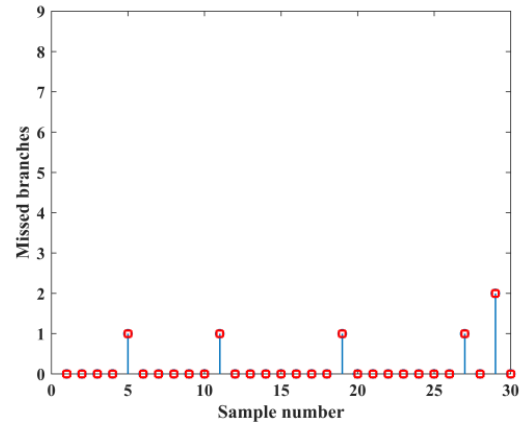

(b)

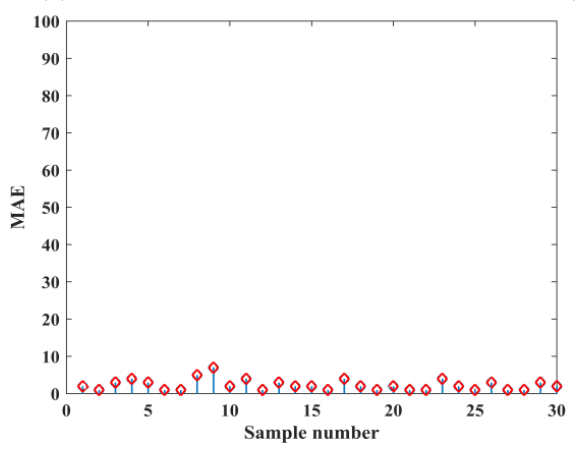

(c)

Fig. 8. Different measurement scores of the sample images: (a) is the number of over-detected branches of the cracks (fake information); (b) is the number of the missed branches of the detected results; (c) MAE of the proposed methods

To fully demonstrate the performance of the proposed algorithm, more sample images are adopted including the ones of Fig. 7. The results of Fig. 8a and b should be discussed together at the same iteration time with the same parameters set, and to reduce the amount of missing branches which may result in unexpected consequence as less as possible, the algorithm achieves the result of only $6 \%$ possibility of missing detection with respect to the rate of $38 \%$ unexpected branches. On the other hand, the MAE result is extremely well with the criteria that the accuracy loss of MAE could 
be ignored when the highest score is less than 10 pixels [33]. And the accuracy rate of (a) and (b) could be improved considerably by increasing the times of iteration.

\subsection{Comparison with state-of-the-art methods}

In this section, other three classical crack detection frameworksincluding the threshold-based segmentation model [50], clustering model [20], region growing [27] are introduced to perform the crack detection on different kinds of sample images to demonstrate the efficiacy of the proposed algorithm. The results correponding to the three groups of Fig. 8 are shown in Fig. 9.

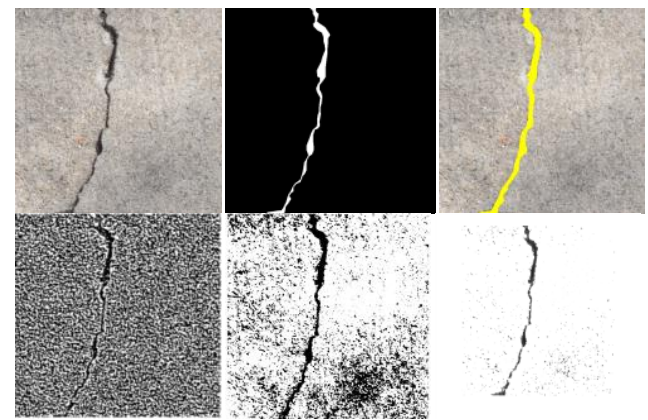

Group A: Images with single cracks

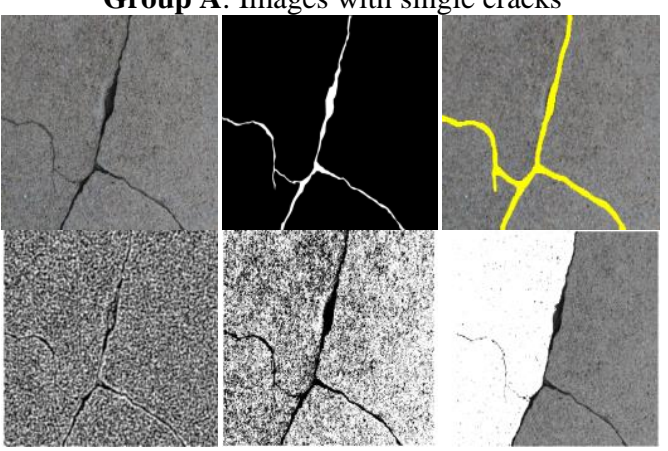

Group B: Images with branches

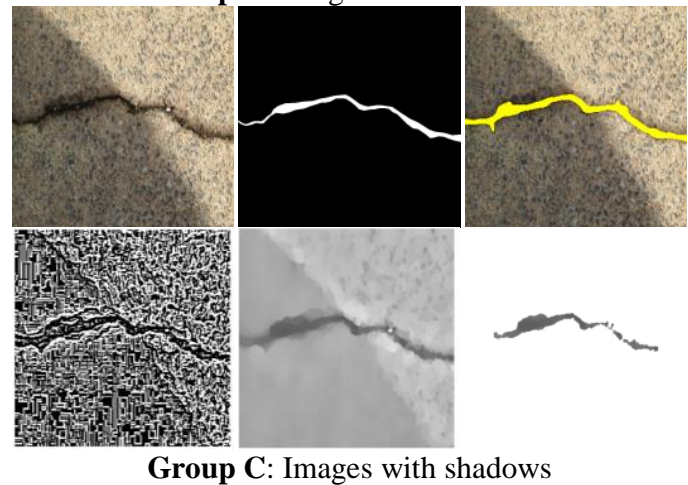

Fig. 9. Crack detection result comparison with other three state-of-the-art detection framework. In each group, the first one is the original image, the second one is the ground truth image, the third one is the result by the proposed algorithm, the fourth one is processed by OTSU, while the fifth and sixth one is obtained by $\mathrm{K}$-means and region growing processing, and the process of Group $\mathrm{C}$ is firstly conducted the shadow-removal procedure 
It is obvious that the detection result by the proposed algorithm is far better than the other three models. The result by the OTSU is of no significance because the SNR ratio is so low that it could not be adopted for practical diagnosis of cracks on infrastructures. The result of k-means calls for further process to remove the black parts (useful information of binarized result) defined as noise information. As for the result by region growing method, it could be regarded as an efficient framework based on the results in Group A and Group C images while the result of Group B is out of expectation. However, the detection accuracy of the result is still far away from that of the proposed methods. What's more, it is unnecessary to analyze the proposed measurement score because the result is easy to analyze from the perspective of visual information.

\section{Conclusion}

In this paper, we have presented a unified 'neuron segmentation' framework for detecting various cracks from the cluttered infrastructural background. Automated initialization of the level set without human intervention on seed point selection is performed by adopting Otsu's global thresholding theory followed by primary noise removal using morphological open operators; the final level set is obtained by using binary distance transform. Then cracks segmentation is fulfilled with an integrated fashion by incorporating the directional region growing using the generated level sets. The design of crack evidence generation based on local directional filtering for replacing the original hessian matrix one in traditional TuFF enables the model join the existing fragments without importing fake crack information efficiently and accurately. Experimental results demonstrate that the cracks detected by the proposed algorithm are very similar to the expert-manually traced ground truth ones.

However, the proposed crack segmentation using TuFF with local directional evidence still has the disadvantage of high time consumption because the detection is performed iteratively, and it should be mentioned that in our implementation we have focus more on the segmentation accuracy at the cost than on making the algorithm run faster. In the future, we will focus on the global thresholding part and the numerical PDE evolution part to reduce the computational time consumption. What's more, designing a robust algorithm to realize the cracks' width calculation followed by the detection procedure is essential to publish a holonomic and automated crack processing framework for practical purpose.

Acknowledgements: The research described in this paper has been supported financially by Jiangsu Collaborative Innovation Center for Building Energy Saving and Construct Technology (Grant No SJXTY1518).

\section{References}

1. National Transportation Safety Board, Collapse of 1-35W Highway Bridge, Minneapolis, 1 August 2007.

http://www.dot.state.mn.us/i35wbridge/ntsb/finalreport.pdf

2. A s a ku ra, T., Y. K o j i ma. Tunnel Maintenance in Japan. - Tunnelling \& Underground Space Technology, Vol. 18, 2003, No 2-3, pp. 161-169. 
3. K o c h, C., K. G e or g i e va, V. K a s i r e d d y et al. A Review on Computer Vision Based Defect Detection and Condition Assessment of Concrete and Asphalt Civil Infrastructure. - Advanced Engineering Informatics, Vol. 29, 2015, No 2, pp. 196-210.

4. $\mathrm{Z}$ a u r i n, R., F. N. C a t b a s. Integration of Computer Imaging and Sensor Data for Structural Health Monitoring of Bridges. - Smart Materials \& Structures, Vol. 19, 2009, No 1, pp. 427-437.

5. P a d e n, A., T. B r y a n t, D. E. L u et al. Supervised Computer-Vision-Based Sensing of Concrete Bridges for Crack-Detection and Assessment. - Transportation Research Board Annual Meeting, 2014.

6. J ah anshah i, M. R., J. S. Kelly, S. F. M a s ri et al. A Survey and Evaluation of Promising Approaches for Automatic Image-Based Defect Detection of Bridge Structures. - Structure \& Infrastructure Engineering Maintenance, Vol. 5, 2009, No 6, pp. 455-486.

7. A bde lq a d e r, I., O. A bud a y e h, M. E. Ke 11 y. Analysis of Edge-Detection Techniques for Crack Identification in Bridges. - Journal of Computing in Civil Engineering, Vol. 17, 2014, No 4, pp. 255-263.

8. Non-Member T. Y., Non-Member S. N., Non-Member R. S. et al. Image-Based Crack Detection for Real Concrete Surfaces. - IEEE Transactions on Electrical \& Electronic Engineering, Vol. 3, 2008, No 1, pp. 128-135.

9. Y a m a g u c h i, T., S. H a s h i m o t o. Fast Crack Detection Method for Large-Size Concrete Surface Images Using Percolation-Based Image Processing. - Machine Vision \& Applications, Vol. 21, 2010, No 5, pp. 797-809.

10. Koch, C., S. G. P a a l, A. R a shidi et al. Achievements and Challenges in Machine VisionBased Inspection of Large Concrete Structures. - Advances in Structural Engineering, Vol. 17, 2014, No 3, pp. 303-318.

11. S i n h a, S. K., P. W. F i e g u th. Automated Detection of Cracks in Buried Concrete Pipe Images. - Automation in Construction, Vol. 15, 2006, No 1, pp. 58-72.

12. S i n h a, S. K., P. W. F i e g u th. Morphological Segmentation and Classification of Underground Pipe Images. - Machine Vision \& Applications, Vol. 17, 2006, No 1, pp. 21-31.

13. F u j it a, Y., Y. H a m a m o t o. A Robust Automatic Crack Detection Method from Noisy Concrete Surfaces. - Machine Vision \& Applications, Vol. 22, 2011, No 2, pp. 245-254.

14. A b d e lqad e r, I., O. A b u d a y y e h, M. E. K e 11 y. Analysis of Edge-Detection Techniques for Crack Identification in Bridges. - Journal of Computing in Civil Engineering, Vol. 17, 2014, No 4, pp. 255-263.

15. Ch a m b o n, S., P. S u b i r a t s, J. Du m o u lin. Introduction of a Wavelet Transform Based on 2D Matched Filter in a Markov Random Field for Fine Structure Extraction: Application on Road Crack Detection. - In: Proc. of SPIE - The International Society for Optical Engineering, 2009.

16. Li, Q., Q. Z o u, D. Z h a n g. FoSA: F* Seed-Growing Approach for Crack-Line Detection from Pavement Images. - Image \& Vision Computing, Vol. 29, 2011, No 12, pp. 861-872.

17. N g u y e n, T. S., S. B e g o t, F. D u c u 1 t y et al. Free-Form Anisotropy: A New Method for Crack Detection on Pavement Surface Images. - In: 18th IEEE International Conference on Image Processing (ICIP), 2011, pp. 1069-1072.

18. A b d e l-Q a d e r, I., S. P a s h a i e-R a d, O. A b u d a y y e h, S. Y e h i a. PCA-Based Algorithm for Unsupervised Bridge Crack Detection. - Adv. Engineering Software, Vol. 37, 2006, No 12 , pp. 71-778.

19. Pras an n a, P., K. D a n a, N. Gu c un ski et al. Computer-Vision Based Crack Detection and Analysis. - Sensors \& Smart Structures Technologies for Civil Mechanical \& Aerospace Systems, 2012, 8345 .

20. Lattanzi, D., G. R. Mil1e r. Robust Automated Concrete Damage Detection Algorithms for Field Applications. - Journal of Computing in Civil Engineering, Vol. 28, 2014, No 2, pp. 253-262.

21. Y u, S. N., J. H. J a n g, C. S. H a n. Auto Inspection System Using a Mobile Robot for Detecting Concrete Cracks in a Tunnel. - Automation in Construction, Vol. 16, 2007, No 3, pp. 255-261.

22. G u n k e l, C., A. S t e p p e r, A. C. M ü 11 e r et al. Micro Crack Detection with Dijkstra's Shortest Path Algorithm. - Machine Vision \& Applications, Vol. 23, 2012, No 3, pp. 589-601.

23. Vive k, K., Y. A n th on y, J. T. Y i ch a n g. Detecting Curves with Unknown Endpoints and Arbitrary Topology Using Minimal Paths. - IEEE Transactions on Software Engineering, Vol. 36, 2011, No 3, pp. 1952-1965.

24. Ts a i, Y., V. Kau l, A. Y ezzi. Automating the Crack Map Detection Process for Machine Operated Crack Sealer. - Automation in Construction, Vol. 31, 2013, No 3, pp. 10-18.

25. Z h a o, G., T. W a n g, J. Y e. Surface Shape Recognition Method for Crack Detection. - Journal of Electronic Imaging, Vol. 23, 2014, No 3, pp. 6-8.

26. Z o u, Q., Y. C a o, Q. Li et al. Crack Tree: Automatic Crack Detection from Pavement Images. Pattern Recognition Letters, Vol. 33, 2012, No 3, pp. 227-238. 
27. X i e, J., T. Z h a o, T. L e e et al. Anisotropic Path Searching for Automatic Neuron Reconstruction. - Medical Image Analysis, Vol. 15, 2011, No 5, pp. 680-689.

28. P en g, H., F. L o n g, G. M y ers. Automatic 3D Neuron Tracing Using All-Path Pruning. Bioinformatics, Vol. 27, 2011, No 13, pp. i239-i247.

29. D i j k s t r a, B. E. A Note on Two Problems in Connection with Graphs. - Numerische Mathematics, Vol. 1, 1959, No 1, pp. 269-271.

30. Jahan shahi, M. R., S. F. Masri, C. W. Padgett et al. An Innovative Methodology for Detection and Quantification of Cracks through Incorporation of Depth Perception. - Machine Vision \& Applications, Vol. 24, 2013, No 2, pp. 227-241.

31. Jah an sh ah i, M. R., S. F. M a s ri. Adaptive Vision-Based Crack Detection Using 3D Scene Reconstruction for Condition Assessment of Structures. - Automation in Construction, Vol. 22, 2012, pp. 567-576.

32. Toro k, M. M., M. G ol par var-Fard, K. B. Ko che r s berger. Image-Based Automated 3D Crack Detection for Post-Disaster Building Assessment. - Journal of Computing in Civil Engineering, Vol. 28, 2013.

33. M u k h e r j e e, S., C. B a r r y, S. T. A c t o n. Tubularity Flow Field - A Technique for Automatic Neuron Segmentation. - IEEE Transactions on Image Processing, Vol. 24, 2015, No 1, pp. 374-389.

34. A l-Kofahi, K. A., C. A li, L. Sh arie et al. Median-Based Robust Algorithms for Tracing Neurons from Noisy Confocal Microscope Images. - IEEE Transactions on Information Technology in Biomedicine, Vol. 7, 2003, No 4, pp. 302-317.

35. Rodrigue z, A., D. B. Ehl e n b e rg e r, P. R. Hof et al. Three-Dimensional Neuron Tracing by Voxel Scooping. - Journal of Neuroscience Methods, Vol. 184, 2009, No 1, pp. 169-175.

36. W e a r n e, S. L., A. R o d ri g u e z, D. B. E h l e n b e r g e r et al. New Techniques for Imaging, Digitization and Analysis of Three-Dimensional Neural Morphology on Multiple Scales. Neuroscience, Vol. 136, 2005, No 3, pp. 661-680.

37. Cun tz, H., F. Forstner, J. Ha g et al. The Morphological Identity of Insect Dendrites. Plos Computational Biology, Vol. 4, 2009, No 12, pp. 1271-1276.

38. M u k h e r j e e, S., S. T. A c t o n. Vector Field Convolution Medialness Applied to Neuron Tracing. - In: 20th IEEE International Conference on Image Processing (ICIP'13), 2013, IEEE, pp. 665-669.

39. B a s u, S., B. C o n d r o n, A. A k s e 1 et al. Segmentation and Tracing of Single Neurons from 3D Confocal Microscope Images. - IEEE Transactions on Information Technology in Biomedicine, Vol. 17, 2012, No 2, pp. 319-335.

40. Mukherjee, S., S. B as u, B. Condron et al. Tree2Tree2: Neuron Tracing in 3D. International Symposium on Biomedical Imaging, 2013, pp. 448-451.

41. V e s e, L. A., T. F. C h a n. A Multiphase Level Set Framework for Image Segmentation Using the Mumford and Shah Model. - International Journal of Computer Vision, Vol. 50, 2002, No 3, pp. 271-293.

42. Z h a o, H. K., T. Ch a n, B. M e r r i m a n et al. A Variational Level Set Approach to Multiphase Motion. - Journal of Computational Physics, Vol. 127, 1996, No 1, pp. 179-195.

43. Ot s u, N. A Threshold Selection Method from Gray-Level Histograms. - Automatica, Vol. 11, 1975, Nos. 285-296, pp. 23-27.

44. A c t o n, S. T. Fast Algorithms for Area Morphology. - Digital Signal Processing, Vol. 11, 2001, No 3, pp. 187-203.

45. J a c o b, M., M. Un s e r. Design of Steerable Filters for Feature Detection Using Canny-Like Criteria. - IEEE Transactions on Pattern Analysis and Machine Intelligence, Vol. 26, 2004, No 8, pp. 1007-1019.

46. S t a n l e y, O., J. A. S e th i a n. Fronts Propagating with Curvature-Dependent Speed: Algorithms Based on Halmiton-Jacobi Formulations. - Journal of Computational Physics, Vol. 79, 1988, No 1, pp. 12-49. 